\title{
Effet du billonnage, de la densité de plantation et de la fumure organique sur la production de racines tubérisées de Vernonia kotschyana Sch. Bip. ex Walp
}

\author{
Seydou Mamadou DEMBELE ${ }^{2 *}$, Mamadou Lamine DIARRA ${ }^{1}$, Ngolo BALLO $^{2}$, \\ Mahamane HAIDARA ${ }^{1}$, Adama DENOU ${ }^{1}$, Rokia SANOGO $^{1,2}$ et Drissa DIALLO ${ }^{1,2}$ \\ ${ }^{1}$ Faculté de Pharmacie, Université des Sciences, des Techniques et des Technologies de Bamako, BP.1805, \\ Bamako, Mali. \\ ${ }^{2}$ Département de Médecine Traditionnelle (DMT), Institut National de Recherche en Santé Publique (INRSP), \\ BP.1746, Bamako, Mali. \\ *Auteur correspondant ; E-mail: seydoumamadoudembele@yahoo.fr /Tel :(+223)76281990.
}

\section{RESUME}

Au Mali, les racines tubérisées de Vernonia kotschyana Sch. Bip. ex Walp. sont utilisées pour le traitement des gastrites et de l'ulcère gastroduodénal. La pression d'exploitation de la plante a entrainé la raréfaction de cette plante dans la nature. Dans le but de l'introduire en culture, un essai de culture a été entrepris. Pour ce faire, l'effet de la technique de travail du sol, de la fumure organique et de la densité de plantation sur la production de racines tubérisées a été étudié à travers un dispositif en Split-plot en 4 répétitions. Les résultats obtenus ont montré que la production de racines tubérisées chez Vernonia kotschyana est influencée par la technique de travail du sol $(6,30 \mathrm{t} / \mathrm{ha})$ et la densité de plantation $(8,25 \mathrm{t} / \mathrm{ha})$. Par contre, malgré une production maximale de $8 \mathrm{t} / \mathrm{ha}$, la fumure organique n'a pas produit d'effet significatif sur la production de racines tubérisées. Toutefois, l'interaction densité de plantation/dose d'engrais a produit des effets significatifs sur la production de racines tubérisées. En conclusion, le billonnage et la densité de plantation ont produit le meilleur effet sur la production de racines tubérisées.

(C) 2019 International Formulae Group. All rights reserved.

Mots clés: Vernonia kotschyana, culture, production de racines tubérisées.

\section{Ridging effect, planting density and organic manure on the production of tuberous roots of Vernonia kotschyana Sch. Beep. ex Walp.}

\begin{abstract}
In Mali, the tuberous roots of Vernonia kotschyana Sch. Beep. ex Walp. are used for the treatment of gastritis and peptic ulcer. The operating pressure of the plant has led to the rarefaction of this plant in nature. With a view to introduce it into culture, a culture test was undertaken. To do this, the effect of the tillage technique, organic manure and planting density on the production of tuberous roots was studied through a splitplot device in 4 repetitions. The results obtained showed that the production of tuberous roots in Vernonia kotschyana is influenced by tillage technique $(6.30 \mathrm{t} / \mathrm{ha})$ and planting density $(8.25 \mathrm{t} / \mathrm{ha})$. On the other hand, despite a maximum production of $8 \mathrm{t} / \mathrm{ha}$, the organic manure did not have a significant effect on the production of tuberous roots. However, the interaction planting density/fertilizer dose produced significant effects on the
\end{abstract}


production of tuberous roots. In conclusion, ridging and planting density produced the best effect on tuberous root production.

(C) 2019 International Formulae Group. All rights reserved.

Keywords: Vernonia kotschyana, culture, tuberous root production.

\section{INTRODUCTION}

Un médicament traditionnel amélioré est un médicament produit à base de plantes issues des pharmacopées traditionnelles, de composition chimique testée, qui a fait l'objet de tests de toxicité sur les animaux, dont les études scientifiques ont évalué l'efficacité thérapeutique et dont la production est contrôlée (Pousset, 2006). La médecine traditionnelle occupe une place de choix dans la couverture sanitaire du pays à l'instar des autres pays Africains où selon l'OMS, « $80 \%$ de la population africaine utilisent la médecine traditionnelle à base des plantes médicinales pour les soins de santé primaire ».

$\mathrm{Au}$ Mali, les ulcères gastroduodénaux et les gastrites connaissent une généralisation plus ou moins grande depuis quelques années (Keita, 2005). Ils sont traités par les racines tubérisées de Vernonia kotschyana. Un médicament traditionnel amélioré appelé Gastrosédal est préparé à partir des racines tubérisées de Vernonia kotschyana (Ministère de la Santé du Mali, 2005). La forte pression d'exploitation de Vernonia kotschyana pose des problèmes concrets de sa dégradation se traduisant par des pénuries de matières premières (racines tubérisées) pour la production de médicament traditionnel amélioré et la réduction progressive de son habitat. La seule véritable solution pour assurer la disponibilité de matière première des plantes médicinales est leur culture. Le choix de la technique culturale utilisée peut influencer la production de matière première. (Fennell et al., 2004). Les plantes médicinales cultivées présentent un certain nombre d'avantages tel que la qualité de la matière première, le séchage effectué dans de bonnes conditions, le traitement du matériel végétal au voisinage du champ supprimant toute altération des drogues pendant le transport etc. (Rubin, 2004). Ainsi, pour assurer un approvisionnement régulier en racines tubérisées, cette étude a été entreprise avec comme objectif général d'identifier les conditions optimales de culture de Vernonia kotschyana en vue d'obtenir une production plus accrue.

\section{MATERIEL ET METHODES}

L'essai a été conduit à Sotuba $\left(12^{\circ} 39^{\prime} 44.1^{\prime \prime} \mathrm{N}\right.$ et $\left.7^{\circ} 57^{\prime} 10.6^{\prime \prime} \mathrm{W}\right)$ en commune I du district de Bamako au cours de la campagne agricole 2007-2008. Sotuba est situé dans la zone soudano-sahélienne avec une pluviométrie moyenne annuelle de 890,5 $\mathrm{mm}$. La saison des pluies y dure 06 mois. L'amplitude thermique annuelle est de $24,5{ }^{\circ} \mathrm{C}$ avec des températures moyennes extrêmes variant de $39,5 \quad{ }^{\circ} \mathrm{C}$ à $15 \quad{ }^{\circ} \mathrm{C}$ (Dembélé, 2009). Le matériel végétal est constitué de plants de Vernonia kotschyana issus de semis direct de graines. La fumure organique était constituée par la bouse de vache.

Le dispositif expérimental est un splitplot. Les facteurs étudiés étaient la technique de travail du sol, la densité de plantation et la dose de fumure organique. Trois niveaux ont été utilisés pour la densité: E1 (57 plants par sous parcelle), E2 (30 plants par sous parcelle et E3 (15 plants par sous parcelle), soit respectivement 95000,50000 et 25000 plants par hectare. En vue de ne pas dépasser la dose économique, nous avons procédé progressivement par de petites doses de fumure organique (00 t/ha, $04 \mathrm{t} / \mathrm{ha}$ et $08 \mathrm{t} / \mathrm{ha})$. Le dispositif a été implanté en 04 répétitions. Le billonnage et son témoin étaient les traitements principaux, les traitements secondaires étaient constitués par l'interaction entre la densité de plantation et les niveaux d'apport de la fumure organique. Tous les traitements ont été affectés au hasard aux parcelles principales et aux sous parcelles 
individuellement à l'intérieur de chaque bloc. Les graines ont été semées directement dans les poquets et les plants ont été démariés à une semaine après la levée. Le désherbage a été fait en deux passages suivant le niveau d'enherbement des parcelles.

Les récoltes ont été faites 05 mois après la levée. Le rendement en racines tubérisées fraiches a été le paramètre mesuré.

\section{Analyses statistiques}

Les données ont été traitées avec le logiciel XLSTAT PRO 7.1. Le test de Newman et Keuls au seuil de signification de $5 \%$, a été utilisé pour la séparation des moyennes.

\section{RESULTATS}

Les résultats de la technique de travail du sol, de la densité de plantation, de la dose de fumure organique et de l'interaction densité de plantation/dose de fumure organique sur la production de racines tubérisées fraiches sont présentés dans le Tableau 1.

Ce résultat nous montre que les billons ont enregistré en moyenne une production de racines tubérisées $(6,30 \mathrm{t} / \mathrm{ha})$ plus élevée que celle des parcelles témoins $(3,87 \mathrm{t} / \mathrm{ha})$. Les productions moyennes de racines tubérisées augmentent avec la densité de plantation $(8,25$ t/ha) et la dose de fumure organique $(7,30$ t/ha). La production maximale de racines tubérisées par interaction a été obtenue avec la combinaison densité de plantation la plus élevée/dose maximale de fumure organique (7,68 t/ha).

Il ressort aussi du Tableau 1 qu'une différence significative a été observée avec la technique de travail du sol, la densité de plantation et la combinaison densité de plantation/ dose de fumure organique. Par contre la fumure organique n'a produit aucun effet significatif sur la production de racines tubérisées.

Les résultats de la séparation des moyennes de production de racines tubérisées fraiches en fonction de la technique de travail du sol, de la densité de plantation, de l'interaction densité de plantation/dose de fumure organique sont présentés dans le Tableau 2.

Ce tableau nous montre la présence de 6 groupes de moyennes homogènes $(\mathrm{A}, \mathrm{B}$, $\mathrm{AB}, \mathrm{ABC}, \mathrm{BC}$ et $\mathrm{C})$.

Par la technique de travail du sol, le billonnage a produit le meilleur effet sur la production de racines tubérisées fraiches $(6,30$ t/ha).

La densité de plantation de 95000 plants par hectare (E1) a produit le meilleur effet sur la production de racines tubérisées fraiches $(6,62 \mathrm{t} / \mathrm{ha})$.

L'interaction densité de plantation/dose de fumure organique a produit le meilleur effet sur la production de racines tubérisées fraiches $(7,68 \mathrm{t} / \mathrm{ha})$ avec la combinaison plus forte densité/plus forte dose (95 000 plants/ha/D3).

Tableau 1: Production moyenne de racines tubérisées fraiches de Vernonia kotschyana en fonction de la technique de travail du sol, de la densité de plantation, de la dose de fumure organique et de l'interaction densité de plantation/dose de fumure organique.

\begin{tabular}{llllc}
\hline Facteurs & & $\begin{array}{l}\text { Productions } \\
\text { moyennes (t/ha) }\end{array}$ & Signification & Ecart-type \\
\hline Technique de travail du sol & Billonnage & 06,30 & $\mathrm{~S}$ & 2,88 \\
& Témoin & 03,87 & 1,84 \\
\hline $\begin{array}{l}\text { Densité de plantation par } \\
\text { hectare }\end{array}$ & E1 (95 000 plants) & 08,25 & $\mathrm{~S}$ & 2,83 \\
& E2 (50 000 plants) & 06,11 & 2,61 \\
& E3 (25 000 plants) & 04,53 & 1,76 \\
\hline
\end{tabular}




\begin{tabular}{|c|c|c|c|c|}
\hline \multirow[t]{3}{*}{ Dose de fumure organique } & D0 $(00 \mathrm{t})$ & 05,27 & \multirow{3}{*}{ NS } & 1,98 \\
\hline & $\mathrm{D} 2(04 \mathrm{t})$ & 06,33 & & 2,82 \\
\hline & D3 (08 t) & 07,30 & & 3,31 \\
\hline \multirow{9}{*}{$\begin{array}{l}\text { Interaction densité/dose de } \\
\text { fumure organique }\end{array}$} & E1D0 & 05,08 & \multirow{9}{*}{$S$} & 1,66 \\
\hline & E1D2 & 07,00 & & 2,69 \\
\hline & E1D3 & 07,68 & & 3,17 \\
\hline & E2D0 & 04,34 & & 1,81 \\
\hline & E2D2 & 04,42 & & 2,38 \\
\hline & E2D3 & 06,31 & & 2,75 \\
\hline & E3D0 & 03,12 & & 1,81 \\
\hline & E3D2 & 03,91 & & 1,73 \\
\hline & E3D3 & 03,87 & & 1,68 \\
\hline
\end{tabular}

$\mathrm{S}:$ Significatif ; NS : Non significatif ; $\mathrm{t}$ : tonne ; ha : hectare.

Tableau 2 : Séparation des moyennes de production de racines tubérisées fraiches en fonction de la technique de travail du sol, de la densité de plantation, de l'interaction densité de plantation/dose de fumure organique.

\begin{tabular}{|c|c|c|c|}
\hline Facteurs & & Groupes homogènes & Ecart-type \\
\hline \multirow[t]{2}{*}{ Technique de travail du sol } & Billonnage & $\mathrm{A}$ & 2,72 \\
\hline & Témoin & B & \\
\hline \multirow{4}{*}{$\begin{array}{l}\text { Densité de plantation par } \\
\text { hectare }\end{array}$} & E1 (95 000 plants) & A & 270 \\
\hline & E2 (50 000 plants) & B & \\
\hline & E3 (25 000 plants) & B & \\
\hline & E1D0 & A & \\
\hline \multirow{8}{*}{$\begin{array}{l}\text { Interaction densité/dose } \\
\text { fumure organique }\end{array}$} & E1D2 & $\mathrm{AB}$ & \\
\hline & E1D3 & $\mathrm{ABC}$ & 2,72 \\
\hline & E2D0 & $\mathrm{ABC}$ & \\
\hline & E2D2 & $\mathrm{ABC}$ & \\
\hline & E2D3 & $\mathrm{ABC}$ & \\
\hline & E3D0 & $\mathrm{BC}$ & \\
\hline & E3D2 & $\mathrm{BC}$ & \\
\hline & E3D3 & $\mathrm{C}$ & \\
\hline
\end{tabular}




\section{DISCUSSION}

La fumure organique n'a pas montré d'effet significatif sur la production de racines tubérisées de Vernonia kotschyana. Un résultat similaire a été signalé par Diarra à 150 jours après repiquage (Diarra et al., 2018) avec Vernonia kotschyana. D'autre part la production de racines de Chlorophytum borivilianum, de Panax ginseng et de Pimpinellae peregrina n'a pas été influencée significativement par la fertilisation (Kothari et Singh, 2003 ; Zhang et al., 2013 ; Carron et al., 2015). La production maximale avec la fumure organique a été obtenue avec la dose maximale. Diarra a obtenu un résultat similaire en 2018. La production de racines tubérisées a été significative avec la densité de plantation. D'autres auteurs ont obtenu ce même résultat avec Chlorophytum borivilianum, Panax ginseng, Pimpinellae peregrina (Kothari et Singh, 2003 ; Zhang et al., 2013 ; Carron et al., 2015). La production de racines tubérisées la plus forte avec interaction a été obtenue avec la combinaison densité de plantation plus élevée/dose de fumure organique maximale (7,68 t/ha). Ce résultat est différent de celui de Carron et al. (2015), où la plus forte production de racines tubérisées de Pimpinellae peregrina (241 $\mathrm{g} / \mathrm{m}^{2}$ ) avec interaction a été obtenue avec la combinaison densité de plantation plus faible/dose de fumure organique plus élevée (Carron et al., 2015).

\section{Conclusion}

Il ressort de cette étude que la technique culturale influe sur la production de racines tubérisées de Vernonia kotschyana. Dans les conditions de cette étude, la production croit significativement avec le billonnage, la densité de plantation et l'interaction densité de plantation/dose de fumure organique. Pour une bonne production de racines tubérisées de Vernonia kotschyana, nous recommandons de cultiver sur billon à la densité de 95000 plants/ha en apportant de la fumure organique.

\section{CONFLIT D'INTERETS}

Les auteurs déclarent qu'il n'y a aucun conflit d'intérêts pour cet article.

\section{CONTRIBUTIONS DES AUTEURS}

SMD a conçu, conduit l'étude et participé à la rédaction de l'article. MLD, NB, $\mathrm{AD}$ et $\mathrm{MH}$ ont participé à la rédaction de l'article. RS, et DD ont relu et corrigé l'article.

\section{REMERCIEMENTS}

Les auteurs remercient le personnel du Département de Médecine Traditionnelle de l'Institut National de Recherche en Santé Publique.

\section{REFERENCES}

Carron CA, Kindlovits S, Baroffio C, Carlen C. 2015. Densité de semis et date de récolte: effet sur le rendement et la qualité de Pimpinella peregrina L. Revue suisse Viticulture, Arboriculture, Horticulture, 47 (5): 280-288.

Dembélé S. 2009. La réponse physiologique de Vernonia kotschyana (Sch. Bip.) à trois techniques culturales : le billonnage, la densité de peuplement et la dose d'engrais. Mémoire de D.E.A. Université de Ouagadougou, Ouagadougou, p. 49.

Diarra ML, Dénou A, Coulibaly BL, Togola A, Sanogo R, Traoré M, Diallo D, Noba K. 2018. Caractéristiques botaniques et phytochimiques de Vernonia kotschyana Sch. Bip. ex Walp. mise en culture et utilisée dans le traitement des gastrites et l'ulcère gastroduodénal au Mali. International Journal of Biological and Chemical Sciences, 12(1): 381-391. DOI: https://dx.doi.org/10.4314/ijbcs.v12i1.30 
Fennell CW, Light ME, Sparg SG, Stafford GI, Staden J Van. 2004. Assessing African medicinal plants for efficacy and safety: agricultural and storage practices. Journal of Ethnopharmacology, 95: 113121. DOI: 10.1016/j.jep.2004.05.025.

Keita A. 2005. Etude de trois plantes utilisées dans le traitement traditionnel de l'ulcère gastro-duodénal dans le district de Bamako : Borassus aethiopum Mart (Palmeae), Sclerocarya birrea (A. Rich). Hochst. (Anacardiaceae) et Ximenia americana L. (Olacaceae). FMPOS, Université de Bamako, Bamako, p. 195.

Kothari S, Singh K. 2003. Production techniques for the cultivation of safed musli (Chlorophytum borivilianum). The Journal of Horticultural Science and
Biotechnology, 78(2): 261-264. DOI: 10.1080/14620316.2003.11511615.

Ministère de la Santé. 2005. Formulaire thérapeutique national, Mali, p. 477.

Pousset JL. 2006. Place des médicaments traditionnels en Afrique. Médecine Tropicale, 66: 606-609.

Rubin M. 2004. GUIDE Pratique de Phytothérapie et d'Aromathérapie. Ellipses : Paris. 6-24.

Zhanga $\mathrm{H}$, Yanga $\mathrm{H}$, Wang $\mathrm{Y}$, Gao $\mathrm{Y}$, Zhang L. 2013. The response of ginseng grown on farmland to foliar-applied iron, zinc, manganese and copper. Industrial Crops and Products, 45: 388-394. DOI: http://dx.doi.org/10.1016/j.indcrop.2012. 12.047 . 